\title{
Reply: Improved safety and effectiveness of imaging predicted for MR mammography
}

\author{
PJ Kneeshaw', LW TurnbulI ${ }^{2}$ and PJ Drew' \\ 'Academic Surgical Unit, Castle Hill Hospital, Castle Road, Cottingham, HUI6 5JQ, UK; ${ }^{2}$ The Centre for Magnetic Resonance Investigations, Hull Royal \\ Infirmary, Hull, UK
}

British Journal of Cancer (2004) 90, 280. doi:I0.1038/sj.bjc.660 I 443 www.bjcancer.com

(c) 2004 Cancer Research UK

Sir,

We read with interest the comments regarding a possible relationship between female carriers of ataxia telangiectasia (AT) with susceptibility to low-dose ionising radiation and the induction of breast cancers in women of screening age.

Although MR mammography does not use ionising radiation, its use in screening of women at a high risk of breast cancer is currently being evaluated, both because of the inherent high sensitivity of the technique, especially in dense pre-menopausal breast tissue and the safety issues particularly in Li-Fraumeni syndrome. The UK Magnetic Resonance Imaging for Breast Screening study (MARIBS) is a multicentre ongoing trial compar-

\section{REFERENCES}

Kriege M, Brekelmans C, Boetes C, Rutgers E, Oosterwijk J, Tollenaar R et al (2003) MRI screening for breast cancer in women with high familial and genetic risk: first results of the Dutch MRI screening study (MRISC). Abstract 5, Cancer Genetics. Presented at the American Society of Clinical Oncology ing the efficacy of X-ray mammography and MRI as a method for screening genetically high-risk women aged $35-50$ years. This trial will close in 2005. Results from Kuhl et al (2003) and Kriege et al (2003) presented recently at the American Society of Clinical Oncology, relating to similar trials again of genetically high-risk subjects have indicated promising results. The authors are unaware of any current trials comparing imaging modalities for screening the 50-64-year age group, or indeed if such a trial is contemplated. The results of such a comparison including health economic issues would need to be very carefully evaluated before embarking on a long-term follow-up trial to elucidate the risks of screening using techniques dependent on ionising radiation.

Kuhl CK, Schrading S, Leutner CC, Morakkabati N, Trog D, Schmutzler R et al (2003) Surveillance of "high risk" women with proven or suspected familial (hereditary) breast cancer: first mid-term results of a multimodality clinical screening trial. Abstract 4 , Cancer Prevention - Early clinical trials. Presented at the American Society of Clinical Oncology 